\title{
Spatial localization of touch in the first year of life: Early influence of a visual code, and the development of remapping across changes in limb position
}

Andrew J. Bremner ${ }^{1,2}$, Denis Mareschal ${ }^{2}$, Sarah Fox ${ }^{2}, \&$ Charles Spence ${ }^{3}$

${ }^{1}$ Department of Psychology, Goldsmiths, University of London

${ }^{2}$ Centre for Brain and Cognitive Development, Birkbeck, University of London

${ }^{3}$ Department of Experimental Psychology, Oxford University

Address for correspondence: Dr Andrew J. Bremner, Department of Psychology, Whitehead Building, Goldsmiths, University of London, New Cross, London, SE14 6NW. United Kingdom. Tel. +44 (0) 207078 5074. Email: a.bremner@gold.ac.uk

Word Count: 9980 (+ Abstract; 165)

References: 85

Running Head: Infants' localization of touch

Keywords: somatosensation, multisensory representation, spatial representation, peripersonal space, perceptual development, infancy.

Acknowledgements: This research was supported by British Academy grant PSRH7 and European Commission grant 516542 (NEST). The authors would like to thank the parents and children who took part in this study, Sue Nicholas and John Pieri for assistance in programming the stimuli and in constructing the testing apparatus and also Ágnes Volein and Megan Crawford for assistance in data collection and coding. 


\begin{abstract}
Two experiments investigated infants' ability to localize tactile sensations in peripersonal space. Ten- (Experiment 1) and 6.5-month-olds (Experiment 2) were presented with vibrotactile stimuli unpredictably to either hand while they adopted either a crossed- or uncrossed-hands posture. At 6.5 months, infants' responses were predominantly manual, whereas at 10 months, visual orienting behavior was more evident. Analyses of the direction of the responses indicated that: i) Both age groups were able to locate tactile stimuli. ii) The ability to remap visual and manual responses to tactile stimuli across postural changes develops between 6.5 and 10 months of age. iii) The 6.5-month-olds were biased to respond manually in the direction appropriate to the more familiar uncrossed-hands posture across both postures. We argue that there is an early visual influence on tactile spatial perception, and suggest that the ability to remap visual and manual directional responses across changes in posture develops between 6.5 and 10 months, most likely due to the experience of crossing the midline gained during this period.
\end{abstract}




\section{Spatial localization of touch in the first year of life: Early influence of a visual code, and the development of remapping across changes in limb position}

Touch provides a very important source of information about the physical environment (e.g., Gregory, 1963). While it is clear that vision provides the richest information about both distal and proximal objects and scenes, it is sufficient to note the prevalence of "do not touch" signs in shops and museums to realize that children (as well as adults) do not rely on vision alone when interacting with the environment. One reason for referring to the tactile sense is that it provides unique information concerning our spatial environment. Touch can only be stimulated by objects in our immediate (or peripersonal) spatial environment. Tactile spatial information is not only useful in its own right, but can also disambiguate which cues from our distal spatial senses (e.g. vision and audition) specify aspects of our peripersonal environment; the environment that affords immediate action. Recent neuroscientific research findings have confirmed this role for touch in peripersonal spatial representation in both human and non-human primates (see Graziano, Gross, Taylor, \& Moore, 2004; Ladavas \& Farnè, 2004; Spence, Pavani, Maravita, \& Holmes, 2004).

Despite the unique spatial information provided by touch, we know relatively little about the development of tactile localization. Instead, research into the early development of spatial abilities has focused primarily on the localization of visually specified objects and features (e.g., Acredolo, 1978; J. G. Bremner, 1978; A. J. Bremner, Bryant, \& Mareschal, 2006; Gilmore \& Johnson, 1997; Kaufman \& Needham, 1999; Mareschal \& Johnson, 2003; Newcombe, Sluzenski, \& Huttenlocher, 2005; Quinn, 1994). Given touch's privileged access to the peripersonal spatial environment, research into the emergence of tactile spatial representation promises, in particular, to shed further light on 
the development of spatial representations for action in the immediate environment. Here we examine localization and crossmodal orienting to tactile stimuli in two age groups; 6.5- and 10-month-old infants. The first year of life is a likely candidate period for developments in tactile localization, and cross-modal orienting to tactile stimulation, as the motor schemas underlying peripersonal exploration develop and integrate substantially between the onset of goal-directed grasping behaviour at around four months of age and before a child's first birthday (e.g., Piaget, 1952; von Hofsten, 2004).

The spatial representation of tactile stimuli is present in some behaviors that are observable at birth and even before. For example, Kisilevsky and Muir (1984) have demonstrated that sleeping neonates will habituate to a brush stroke on either the lips or ear and then dishabituate to brushing at the novel one of these two locations. More recently, Streri and colleagues (Sann \& Streri, 2007; Streri \& Gentaz, 2003, 2004) have demonstrated that newborn infants will habituate and dishabituate (both manually and visually) to different shapes and textures presented to the palms of their hands. The ability to match shape and texture between tactile and visual modalities is thought to be an early acquired skill (Abravanel, 1981; Bryant, Jones, Claxton, \& Perkins, 1972), and has also recently been observed in newborns (Sann \& Streri, 2007; Streri \& Gentaz, 2003, 2004).

Spatial orienting to tactile stimuli is observable in a range of neonatal reflexes. One example of a spatially-specific orienting reflex is observable in newborn crossedextension (Fényes, Gergely, \& Tóth, 1960; Sherrington, 1910; Zappella \& Simopoulos, 1966). Thus, if a newborn infant is touched close to the inguinal canal at the top of the leg they will flex and extend their other leg (Fényes et al., 1960). Neonatal grasping, another orienting response to tactile stimulation, is present even before birth, appearing as early as 11 weeks in utero (see Tan \& Tan, 1999). In addition, Moreau, Helfgott, Weinstein, and 
Milner (1978) have observed the habituation of neonatal head turning to tactile stimulation.

Research indicates that at least some of these early tactile behaviors represent exploratory behaviors rather than just simple transient reflexes (Molina \& Jouen, 1998, 2001; Streri \& Gentaz, 2004), and show effects of laterality which are opposite to those found in childhood (Flanery \& Balling, 1979; Ghent, 1961; Hatta, Yamamoto, Kawabata, \& Tsutui, 1981; Moreau \& Milner, 1981). However, it is important to note that very little is known about the development of tactile orienting responses (either voluntary or involuntary) in infancy beyond the first two months of life. Furthermore, with the possible exception of the study by Moreau et al. (1978), the behaviors described above tell us very little about infants' ability to orient to touch crossmodally. Research into crossmodal orienting responses in infants has focussed almost exclusively on the links between vision and audition. For instance, newborns are known to make saccades (Butterworth \& Castillo, 1976; Wertheimer, 1961) and also head movements (Clifton, Morrongiello, Kulig, \& Dowd, 1981; Muir \& Field, 1979) in the direction of sounds. They also appear to expect visual and auditory stimuli to come from approximately the same location (Aronson \& Rosenbloom, 1971; Morrongiello, Fenwick, \& Chance, 1998). At four months of age, infants will actively orient toward visual stimuli which match an auditory input (Spelke, 1976). Some developments in visual/auditory spatial coordination have also been observed during the first months of life (Bremner, Johnson, Slater, Mason, \& Spring, 2006; Neil, Chee-Ruiter, Scheier, Lewkowicz, \& Shimojo, 2006).

Two early studies have suggested a role for postnatal experience in the development of tactile localization, and/or crossmodal orienting to touch, both during infancy and through middle childhood. Nissen, Chow, and Semmes (1951) examined the role of manual experience in visual and tactile spatial discrimination tasks by severely 
restricting a newborn chimpanzee's tactile, kinaesthetic, visual and manipulative experience with his own hands and feet during the first 30 months of life. This was achieved by fixing restricting cylinders over these limbs. In comparison to another chimpanzee who had received some limited post-natal visual deprivation, the manually deprived chimpanzee demonstrated almost no ability to learn a conditioned head-turn discrimination (i.e., overt orienting response) between two locations stimulated by touch (one on the index finger of either hand).

In another study, Renshaw (1930) developed a task to examine the ability of children and adults to localize punctate tactile stimuli presented on their right arm and hand. The task involved pointing, while blindfolded, to the stimulated locations using the left hand. Interestingly, pre-adolescent children performed better than adults on this task. However, it seems likely that this difference in competence may have been due to developments in the kinds of sensory information which children and adults use when reaching, rather than to any differences in cutaneous spatial acuity per se. Warren and Pick (1970), and indeed Renshaw (1930), have argued that adults rely on visual information for proprioceptive orienting more than do children. Accordingly, the absence of visual information when blindfolded may have provided an advantage for the children in Renshaw's earlier study.

A particular challenge associated with stimuli presented to the tactile modality is the inconsistency in its spatial alignment with the other senses (most especially vision) due to changes in limb position. When the posture of our hands changes, the relation of tactile locations to the visual framework changes, and thus in order to 'relocate' a tactile stimulus location in visual space, a remapping between tactile and visual space is required. Indeed, crossmodal integration more generally faces a problem with accommodating postural change, as the integration of visual with auditory locations 
requires a neural mechanism which can take account of movements of the eyes within their orbits (Pöppel; 1973; Spence, McDonald, \& Driver, 2004). Neurons that remap sensory correspondences across changes in posture have been reported in the monkey superior colliculus (auditory to visual: Jay \& Sparks, 1984; visual to tactile: Groh \& Sparks, 1996b, c) and ventral premotor cortex (visual to tactile: Graziano \& Gross, 1993; Graziano, Yap, \& Gross, 1994; Iriki, Tanaka, \& Iwamura, 1996).

Psychophysical evidence for the spontaneous realignment of the neural representation of stimuli presented to different sensory modalities has, by now, been demonstrated using a number of different psychophysical paradigms, such as the exogenous spatial cuing and crossmodal congruency paradigms (Kennett, Spence, \& Driver, 2002; Spence, McDonald, \& Driver, 2004; Spence, Pavani, \& Driver, 2004). However, a crucial, but as yet unexplored, question concerns how the ability to update crossmodal orienting responses across changes in posture develops in infancy and early childhood.

Thus, as well as investigating the ability of infants to localize tactile stimuli, our investigation also examines early developments in the ability to update the direction of localization responses across changes in the posture of the limbs. In particular, we compared the ability of infants to localize a tactile stimulus across uncrossed-hands and crossed-hands postures. Adoption of the crossed-hands posture changes the mapping of tactile to visual locations such that the visual location that would normally project to the same hemisphere as the tactile stimulus, now projects to the opposite hemisphere. In the uncrossed-hands posture, somatosensory projections from the left hand arrive at the same hemisphere as visual projections from the left side of visual space; both arrive at the contralateral (right) hemisphere. But when the hands are crossed over the midline, the left hand is now situated in the right side of visual external space (and vice versa when the 
right hand is crossed). Tactile stimuli project to the ipsilateral hemisphere, whereas the visual representation of the hand arrives at the contralateral hemisphere (see Spence, Pavani, \& Driver, 2000; Spence, Pavani, Maravita, \& Holmes, 2004). Thus, in order to visually locate touches presented to the crossed hands, some form of interhemispheric spatial remapping is required (see Lloyd, Shore, Spence, \& Calvert, 2003).

Pilot testing of three 10-month-olds' responses to vibrotactile stimuli indicated that these elicited both manual and visual responses. The manual responses took the form of the clenching and unclenching of the fist and/or the withdrawal of the hand by a gross movement of the arm. Visual responses took the form of the visual fixation of the hand that involved eye and/or head movements. Accurate localizations of tactile stimuli by means of these two response modalities in both the uncrossed-hands and crossed-hands postures bear different implications for spatial representation and cross-modal links between touch and vision. In order to localize tactile stimulation with a visual orienting response requires crossmodal links between the representations coding tactile and visual location. Thus, as outlined above, changes in limb position require a remapping of tactile to visual location. However, in order to localize tactile stimuli with a movement of the hand it is possible to rely on a body-centred frame of reference without invoking a visual representation of the location of the hand. Theoretically, using a body-centred frame of reference, it should be possible to make an appropriate manual orienting response toward the stimulated hand across changes in the posture of the arms without having to remap the spatial location in which the response is made.

Nevertheless, a number of recent studies have shown that localization responses to tactile stimulation which do not involve the cross-modal orienting of vision nevertheless do show effects of postural manipulation. For example, Yamamoto and Kitazawa (2001) and Shore, Spry and Spence (2002) have both shown that the accuracy of adult 
participants' temporal order judgments (TOJs) regarding tactile stimuli (one presented to each hand in sequence) are substantially impaired, and sometimes even (inappropriately) reversed when the adult participant has to cross their hands. It has been argued that such postural effects represent the influence of a visual code on spatial perception within the tactile modality (Kitazawa, 2002; Shore et al., 2002).

Röder, Rösler, and Spence (2004) have also presented a striking demonstration that early visual experience may play a key role in establishing this visual influence in tactile spatial perception. Testing sighted, blindfolded sighted, late blind and congenitally blind adults on the tactile TOJ task, they confirmed that sighted participants (no matter whether blindfolded or not) showed substantially less accurate TOJs with crossed hands. However, congenitally blind participants demonstrated no such impairment. Late blind participants (one of whom had been blind for more than 40 years) showed similar crossed-hands impairments to those of the sighted participants, thus suggesting that early visual experience is necessary for the normal development of tactile spatial perception (Eimer, 2004). Converging evidence for the role of vision in tactile localization has also come from two recent studies highlighting a reduction (or even elimination) of the normal hand-crossing effect when people (musicians) crossed their hands behind their backs (i.e., in a region of space that we very rarely, if ever, see; Kóbor, Füredi, Kovács, Spence, \& Vidnyánszky, 2006), and also when sighted participants view a pair of uncrossed rubber hands above their crossed real hands (Azañón \& Soto-Faraco, submitted).

In the experiments reported here, we presented a series of vibrotactile stimuli to the palms of infants' right and left hands. In order to determine whether two age groups of infants (10-month-olds: Experiment 1, and 6.5-month-olds: Experiment 2) were able to remap the location of tactile stimuli across changes in limb position, we compared responses in the uncrossed-hands and crossed-hands postures. In addition to testing the 
ability to remap a visual directional response to tactile location across changes in limb position, the postural manipulation also allowed us to examine whether there is an influence of a visual code on tactile spatial perception. As spontaneous midline crossing in visually directed reaching first appears at around six months of age (Van Hof, Van der Kamp \& Savelsbergh, 2002), the 6.5- and 10-month-old age groups are particularly appropriate for investigating whether remapping of spatial orienting responses across changes in posture bears any relation to midline crossing experience.

\section{Experiment 1}

Experiment 1 was designed to test 10-month-olds' responses to vibrotactile stimuli presented to their left and right palms. Responses were compared across two groups of infants: one adopting an uncrossed-hands posture, and the other adopting a crossed-hands posture ${ }^{1}$

$\underline{\text { Methods }}$

Participants. Fourteen 10-month-olds took part in this study. Usable data were obtained from 12 participants ( 9 boys and 3 girls with a mean age of 302 days; SD $=9.5$ days). The mean age of the 6 infants ( 6 boys) assigned to the Uncrossed-hands group was 306 days $(\mathrm{SD}=9.8)$. The mean age of the 6 infants ( 3 boys, 3 girls $)$ assigned to the Crossed-hands group was 298 days $(\mathrm{SD}=7.4)$. All infants had a gestational age exceeding 37 weeks. The two infants who were excluded from the study did not complete the testing session due to fussy behavior. All testing took place in a specially-designed facility at the university. Ethical approval was gained from the Ethics Committee of the School of Psychology (reference number: 2211) before testing commenced.

Apparatus and stimuli. The experiment was performed in a dimly-lit room, in which the infant's behavior was recorded using an infra-red video camera and VCR. The 
infant was seated on his/her parent's lap with his/her forearms resting on a small table $(51 \mathrm{~cm}$ wide $\times 31 \mathrm{~cm}$ long). Experimenter A faced the infant across the table. One vibrotactile stimulator was placed in the palm of each of the infants' hands, and fixed there with self-adhesive elastic material (Coban). The infants' hands and the stimulators were also covered by small cotton mittens. The vibrotactile stimulators (Oticon bone-

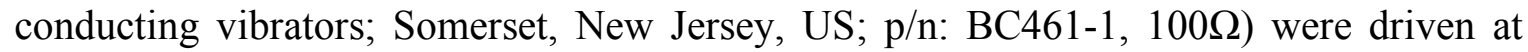
$220 \mathrm{~Hz}$ by a pure tone generator and amplifier. The onset and offset of each stimulator was controlled by means of a custom-built USB-controlled hardware interface which was, in turn, operated by an in-house Visual Basic programme run on a standard personal computer. The software was also set-up to control a serial controlled video titler (Horita SCT-50). Signals that were time-locked with the onset and offset of the vibrotactile stimuli were sent to the video titler such that the infants' stimulus-locked behavior could be observed and recorded.

In order to reduce the likelihood of eliciting directional orienting responses to any subtle sounds elicited by the operation of the tactors, a second pair of masking vibrators was placed on the table just beyond the infant's reach. These vibrators were arranged such that any subtle lateralized auditory information accompanying the vibrotactile stimulus presented to one hand was matched from the other side of the midline by a masking vibrator placed on the table. Quiet music was also played during testing in order to mask the noise of the vibrators. Figure 1 shows an infant taking part in the Crossed-hands and Uncrossed-hands blocks.

\section{--Insert Figure 1 about here--}

Design. All of the infants were presented with one block of ten experimental trials. One group of infants was placed in the uncrossed-hands posture and another in the crossed-hands posture across all 10 trials. A tactile stimulus was administered to the 
infant's left or right hand on each trial. The pseudorandom order in which the hands were stimulated was the same for every infant (R, L, L, L, R, R, L, L, R, R).

Procedure. Before the experimental session, Experimenters A and B greeted the parent and child. Following an explanation of the procedure to the parent, the parent and infant were led through to the testing room. Experimenter A seated the parent and infant at the table, and proceeded to attach the vibrators to the infants' hands and then to cover them with the mittens. Experimenter B was seated out of the infant's sight, observing the infant's behavior on a video monitor. Experimenter B triggered the presentation of the tactile stimuli.

The parent was asked to support the infant at the waist, positioning him/her so that his/her radial midline axis was central and perpendicular to the table, the experimenter, and the video camera, while allowing the infant to move his/her head, eyes, arms and hands.

Once seated with the vibrators in place, Experimenter A familiarized the infant with the structure of a typical trial. This familiarization consisted of holding the infant by each wrist and 'bouncing' their hands three times whilst saying "one, two, three, woo!" This was repeated until the infant appeared to have engaged in this game, and was happy to have his hands moved freely by the experimenter.

Following familiarization, the infant was given 10 test trials in which a vibrotactile stimulus was presented to either their left or right hand. Each trial began with the experimenter initiating the 'bouncing game' to which the infant had been familiarized. In the Uncrossed-hands block, on reaching "woo!", the experimenter placed and held the infant's hands approximately $10 \mathrm{~cm}$ apart, one on either side of the midline. At this point, Experimenter B triggered a vibration to one of the infant's hands. Experimenter A would gently hold the infant's wrists in position until the infant moved them, or else until 8 
seconds had elapsed. All of the infant's visual and manual behaviors were measured during this 8 second period. To avoid influencing the infant's behavior through eyecontact, Experimenter A oriented her face toward the ground. The next trial commenced after eight seconds had elapsed.

The trials in the Crossed-hands block proceeded in exactly the same manner with the exception that Experimenter A would cross the infant's hands just before a vibration was triggered (when Experimenter A said “woo!”). In the Crossed-hands block, just as in the Uncrossed-hands block, the infants' wrists were held roughly $10 \mathrm{~cm}$ apart either side of the midline until the infant moved or the 8 second response period had elapsed. For all infants on all trials the hands were crossed with the right hand on top of the left hand.

Data coding. The direction and latency of infants' first manual and first visual responses to the tactile stimuli were coded from the video records. It was thus possible for infants to be scored for a manual and/or a visual response, or neither response on each trial. A second observer coded a proportion of the total sample. Both observers were aware of the onset and offset of the tactile stimuli, but unaware of their side of presentation (left or right). Stimulus location information was used to calculate the scores after the videos had been coded. Coded directional manual responses comprised any observed movement of one of the hands that was not accompanied by any movement of the other hand. Directional visual responses comprised any observed visual fixation of one of the hands. Responses occurring within 133 milliseconds of the onset of the tactile stimuli were considered as anticipatory and were therefore excluded from the analyses. Inter-observer reliability was obtained from a sample of nine infants (from across both experiments) by examining the number of trial-by-trial agreements between the two observers over whether infants' first manual and visual responses were to the left, right, or 
neither hand. There was $81 \%$ agreement for manual responses, and $88 \%$ for visual responses.

$\underline{\text { Results }}$

--Insert Table 1 about here--

Table 1 displays the mean number of trials in which the 10-month-olds made observable directional manual and/or visual responses within 8 seconds of target onset. A directional response was observed on a mean of 6.83 out of 10 trials. Of these, trial numbers were fairly evenly split between trials on which manual only, visual only or both modalities of response were observed.

--Insert Figure 2 about here--

Figure 2A plots the cumulative number of first manual and visual responses against response latency (sampled at intervals of 500 milliseconds), across all 10 trials. The majority of the first responses occurred within 3 seconds of stimulus onset, and performance levelled off after approximately 5 seconds, reaching the maximum just before 7 seconds. The 10-month-olds made observable visual directional responses on approximately half of the trials and observable manual directional responses on approximately half of the trials as well (all within 8 seconds of target onset). The emergence of manual and visual responses to the tactile stimulus had a very similar temporal profile. In order to determine whether there were any significant differences in the temporal emergence of visual and manual responses to the tactile stimulus, a repeated measures $2 \times 6$ analysis of variance (ANOVA) was conducted on the number of first responses elicited in the two Response modalities (Manual / Visual) sampled at six Latencies in milliseconds after stimulus onset $(250 / 750 / 1250 / 1750 / 2250 / 2750 \mathrm{~ms})$. This analysis revealed a simple main effect of Latency $\left(\underline{\mathrm{F}}(5,55)=38.3, \underline{\mathrm{p}}<.001, \underline{\mathfrak{\eta}}_{p}{ }^{2}=.78\right)$, reflecting the increase in the likelihood of an infant making a response between 250 and 
$2750 \mathrm{~ms}$. No main effect of Response modality nor interaction between Response modality and Latency was observed (both $\underline{F} s<1$ ), indicating that manual and visual responses had a similar timecourse. This is also evident in the average latencies of these responses (shown in the upper left corner of Figure 2A) which did not differ significantly $($ Manual latency $=2.23 \mathrm{~s}$, Visual latency $=1.92 \mathrm{~s} ; \underline{\mathrm{t}}(11)=1.0, \underline{\mathrm{n} . \mathrm{s}} ., \underline{\mathrm{d}}=.3)$.

Manual orienting. Figure 3A (Panel A1) shows the direction (rather than the latency) of infants' first manual responses to the unseen tactile stimuli on each trial in both postures. In the uncrossed-hands posture, the tactile stimulus was presented on the side of visual space contralateral to the cerebral hemisphere that initially received the somatosensory stimulation. In the crossed-hands posture, as the hands changed locations with respect to the midline, the tactile stimulus was now presented on the side of visual space that was ipsilateral to the cerebral hemisphere receiving the somatosensory stimulation. Thus appropriate directional responses are contralateral for the uncrossedhands posture, and ipsilateral for the crossed-hands posture. A 2 × 2 mixed-design ANOVA (Posture group: Uncrossed-hands / Crossed-hands; Response direction: Contralateral / Ipsilateral) of the number of manual responses revealed a significant interaction between Posture and Response direction $\left(\underline{F}(1,10)=17.8, \underline{p}<.005, \underline{\eta}_{p}=.64\right)$. There was no main effect of Response direction or Posture (both $\underline{F}<<1.5$ ). Planned comparisons of responses toward the ipsilateral and contralateral sides were made within each posture. As the Uncrossed-hands posture is presumably that which the infants had most experience with, we predicted robust contralateral responding in this condition. Consequently we used a one-tailed test in this condition, whereas, in the Crossed-hands posture, where we thought it possible that incorrect responding might occur due to habitual contralateral responding, we use a two-tailed test. Infants in the Uncrossed-hands posture made significantly more manual responses to the contralateral side of space 
$(\underline{\mathrm{t}}(5)=2.9$, one-tailed $\mathrm{p}<.02, \underline{\mathrm{d}}=1.2)$, whereas infants in the Crossed-hands posture made significantly more manual responses toward the ipsilateral side $(\mathrm{t}(5)=4.0$, two-tailed $\underline{\mathrm{p}}<.02, \underline{\mathrm{d}}=.8$ ). These results therefore show that ten-month-old infants are able to make appropriate manual orienting responses to tactile stimuli in both postures, and the accuracy of their manual responding is unaffected by their posture (Uncrossed-hands or Crossed-hands).

Visual orienting. Figure 3 (Panel A2) shows the direction of infants' first visual responses to the tactile stimuli on each trial in the Uncrossed-hands and Crossed-hands posture groups. Again, correct localizations are represented by contralateral responses in the Uncrossed-hands posture, and ipsilateral responses in the Crossed-hands posture. A 2 x 2 mixed-design ANOVA (Posture group: Uncrossed-hands / Crossed-hands; Response direction: Contralateral / Ipsilateral) of the number of first visual responses on each trial revealed a significant interaction between Posture and Response direction $(\underline{F}(1,10)=6.0$, $\mathrm{p}<.05, \underline{\eta}_{\mathrm{p}}{ }^{2}=.38$ ), confirming that the direction of infants' visual orienting to the tactile stimuli was influenced by their posture. There were no main effects of Response direction or Posture (both $\underline{F}<<1.5$ ). An inspection of the mean number of responses toward the ipsilateral and contralateral sides within the two posture conditions indicated more contralateral than ipsilateral visual directional responses in the Uncrossed-hands posture, and more ipsilateral than contralateral visual directional responses in the Crossed-hands posture. Planned comparisons of these means failed to reach significance (Uncrossedhands: $\underline{\mathrm{t}}(5)=1.9$, one-tailed $\mathrm{p}=.11, \underline{\mathrm{d}}=.79$; Crossed-hands: $\mathrm{t}(5)=1.7$, two-tailed $\mathrm{p}=.14$, $\underline{\mathrm{d}}=.71$ ). However, given the large effect sizes, this is most likely due to a lack of power within each posture condition. The interaction between posture and response direction, and the large effect sizes within each posture condition suggest that 10-month-olds are able to adapt their visual orienting to tactile stimulation across changes in limb position. 


\section{Discussion}

The 10-month-olds tested here were able to make manual and visual orienting responses toward unseen tactile stimuli presented to their hands, across two different arm postures; a more natural (and more familiar) uncrossed hands posture, and a less familiar crossed-hands posture (see Figure 3A). This is the first demonstration of visual orienting to tactile stimulation in early infancy. Furthermore, manual and visual responses were made appropriately across both familiar (uncrossed-hands) and unfamiliar (crossedhands) arm postures indicating that 10-month-olds are able to orient appropriately following a change in the posture of their hands and arms.

Given these results, we decided to conduct a second experiment in order to determine whether younger infants are also able to update their visual and manual orienting responses to tactile stimulation across changes in posture, using a similar paradigm to that reported in Experiment 1. This allowed us to determine whether there was any early development in tactile localization, and in the ability to orient crossmodally to touch across changes in limb position.

\section{Experiment 2}

Experiment 2 uses the paradigm developed in Experiment 1 to test the localization of tactile stimuli in 6.5-month-olds. A minor procedural modification was made to the paradigm used in Experiment 1 in order to compare postural conditions within these younger infants. Because responses appeared to drop off after the first ten trials in Experiment 1, we reduced the number of trials down to 5 per block in our second experiment.

In addition, a control group of participants was tested on a 'No Stimulation Baseline' (NSB) version of the task in which all aspects of the 'Tactile Stimulation' (TS) 
condition were reproduced except that no tactile stimulation was applied. This control condition was performed in order to compare responses to the vibrotactile stimulation in the Tactile Stimulation condition against baseline levels of manual and visual responses to the task context in crossed- and uncrossed-hands postures. In this No Stimulation Baseline condition, dummy vibrators were placed in the infants' hands and the tactors were activated a short distance from the hands. A second reason to use this control condition was to assess whether the accurate localizations following the stimulation from the vibrators were due to tactile stimulation and not any concurrent auditory information emitted from the vibrators.

Participants. Thirty-two 6.5-month-olds took part in this experiment. Usable data were obtained from 24 participants (11 boys and 13 girls with a mean age of 199 days; SD $=8.3$ days). There were thirteen infants in the Tactile Stimulation group ( 6 boys and 7 girls, with a mean age of 197 days; $\mathrm{SD}=8.8$ days) and 11 in the No Stimulation Baseline group ( 5 boys and 6 girls, with a mean age of 201 days; $\mathrm{SD}=8.8$ days). All infants had a gestational age exceeding 37 weeks. The 8 excluded participants did not complete the testing session due to their fussy behavior.

Design. Infants were assigned to one of two groups: The Tactile Stimulation group who received a tactile stimulus on one hand on each trial, or the No Stimulation Baseline group in which no tactile stimulus was presented but all other aspects of the experimental trials were reproduced.

All of the infants were presented with two blocks of five trials (one block in the Uncrossed-hands posture and the other in the Crossed-hands posture). The order of presentation of the blocks was counterbalanced across infants. The pseudorandom order in which vibration was applied to the left and right hands was the same for all participants 
(Block 1: R, L, L, L, R; Block 2: R, L, L, R, R) regardless of Uncrossed-hands and Crossed-hands block order.

Apparatus and procedure. The apparatus and procedure for the Tactile Stimulation group in Experiment 2 was the same as that used in Experiment 1 (other than those changes of trial order and design mentioned above). In the No Stimulation Baseline condition, two sets of vibrators were placed on the table directly in front of the infants to ensure that the auditory information was matched as closely as possible to that present in the Tactile Stimulation condition. One pair of vibrators was placed in the same position as the auditory masking vibrators used in Experiment 1 and the Tactile Stimulation condition of the current experiment. A second pair of vibrators was placed $3 \mathrm{~cm}$ closer to the infant in order to match the locations occupied by the tactile stimulators in the Tactile Stimulation condition as closely as possible without actually contacting the infants' hands. One vibrator in each pair was activated such that auditory information emanated from both sides on each trial. A pair of dummy vibrators was also placed in the infants' hands, which were again covered with mittens, just as in Experiment 1, and the Tactile Stimulation condition of Experiment 2. The direction of infants' responses in the No Stimulation Baseline condition were scored in relation to the location of the auditory stimulus emanating from the nearest of the two buzzers activated on each trial (i.e. the buzzer simulating the auditory noise that would have emanated from the infant's hand in the Tactile Stimulation group).

$\underline{\text { Results }}$

Table 1 displays the mean number of trials in which the 6.5 -month-olds made observable directional manual and/or visual responses within 8 seconds of target onset. A directional response was observed on a mean of 7.46 out of 10 trials. Predominantly 
manual only responses were observed. Visual responses were much fewer in number in this age group and only very occasionally occurred in isolation.

Response latencies. Figure 2B plots the 6.5-month-olds' cumulative number of first manual and visual responses against response latency relative to stimulus onset (sampled at intervals of $500 \mathrm{~ms}$ ), across all 10 of the administered trials. The majority of the first responses occurred within 3 seconds of stimulus onset, and performance levelled off at a latency of around 4 seconds, reaching the maximum before 6750 milliseconds. Across the 8 second response period, the 6.5-month-olds made about twice as many manual as visual directional responses in both the Tactile Stimulation group (Manual: $\mathrm{M}=7.2, \mathrm{SE}=.58$; Visual: $\mathrm{M}=3.4, \mathrm{SE}=.42$ ) and the No Stimulation Baseline group (Manual: $\mathrm{M}=7.0, \mathrm{SE}=.54$; Visual: $\mathrm{M}=2.7, \mathrm{SE}=.70$ ). A $2 \times 2$ ANOVA of the total number of responses made within the 8s period across Response modality (Manual / Visual) and Group (Tactile Stimulation / No Stimulation Baseline) revealed a significant main effect of Response modality $\left(\underline{\mathrm{F}}(1,22)=58.7, \underline{\mathrm{p}}<.001, \underline{\underline{\eta}}_{p}=.73\right)$. There was no main effect of Group, nor any interaction between Response modality and Group $(\underline{F} s<1)$.

Nonetheless, visual inspection of Figure 2B points to an earlier emergence of manual and visual responses in the No Stimulation Baseline group. We examined any time-locked differences in the emergence of visual and manual responses to the tactile stimuli using a mixed $2 \times 2 \times 6$ ANOVA of the number of first responses elicited in the two Groups (Tactile Stimulation / No Stimulation Baseline), the two Response modalities (Manual / Visual) sampled at six Latencies (250 / 750 / $1250 / 1750$ / 2250 / 2750ms). This analysis revealed a significant main effect of Latency $(\underline{F}(5,110)=49.3, \underline{p}<.001$, $\underline{\underline{\eta}}_{p}{ }^{2}=.69$ ), reflecting the accumulation in the number of first responses elicited between a latency of 250 and 2750 milliseconds, regardless of Response modality or Group. There was also a significant main effect of response modality indicating that the infants made 
fewer visual than manual responses $\left(\underline{\mathrm{F}}(1,22)=27.2, \underline{\mathrm{p}}<.001, \underline{\mathfrak{\eta}}_{p}{ }^{2}=.55\right)$. A significant main effect of Group showed that responses were delayed in the Tactile Stimulation group relative to the No Stimulation Baseline group $\left(\underline{\mathrm{F}}(1,22)=4.2, \underline{\mathrm{p}}=.05, \underline{\underline{\eta}}_{p}=.16\right)$. There was also a significant interaction between Response modality and Latency $((\underline{F}(5,110)=20.5$, $\mathrm{p}<.001, \underline{\mathrm{\eta}}_{p}^{2}=.48$ ), reflecting the increase in the relative number of manual to visual responses after $750 \mathrm{~ms}$ (latency $1250 \mathrm{~ms}$ : $\underline{\mathrm{t}}(23)=2.4, \underline{\mathrm{p}}<.03, \underline{\mathrm{d}}=.48$; latency $1750 \mathrm{~ms}$ : $\underline{\mathrm{t}}(23)=4.4, \underline{\mathrm{p}}<.001, \underline{\mathrm{d}}=.90$; latency $2250 \mathrm{~ms}: \underline{\mathrm{t}}(23)=5.1, \underline{\mathrm{p}}<.001, \underline{\mathrm{d}}=1.0$; latency $2750 \mathrm{~ms}$ : $\underline{\mathrm{t}}(23)=5.8, \underline{\mathrm{p}}<.001, \underline{\mathrm{d}}=1.2)$.

Figure $2 \mathrm{~B}$ also shows the mean latencies of manual and visual responses in the Tactile Stimulation and No Stimulation Baseline groups ${ }^{2}$. These scores were analysed using a 2 x 2 mixed ANOVA (Group: Tactile Stimulation / No Stimulation Baseline; Response modality: Manual / Visual). This analysis revealed a main effect of Response modality with visual responses occurring significantly later than manual responses $\left(\underline{\mathrm{F}}(1,19)=5.4, \underline{\mathrm{p}}<.04, \underline{\underline{\eta}}_{p}=.22\right)$, and an interaction between Group and Response modality $\left(\underline{\mathrm{F}}(1,19)=4.6, \underline{\mathrm{p}}<.05, \underline{\mathrm{\eta}}_{p}^{2}=.19\right)$. There was no main effect of Group $(\underline{\mathrm{F}}<1.5)$. Post-hoc tests revealed that visual responses were made significantly later $(\underline{\alpha}=.025)$ than manual responses in the Tactile Stimulation group (manual latency $=2.81 \mathrm{~s}$, visual latency $=$ $3.71 \mathrm{~s} ; \mathrm{t}(12)=3.4, \mathrm{p}<.004)$ but not in the No Stimulation Baseline group (manual latency $=$ 2.53s. visual latency $=2.81 \mathrm{~s} ; \underline{\mathrm{t}}(8)=.1$, $\underline{\text { n.s. }})$.

--Insert Figure 3 about here--

Manual orienting. Figure 3B displays the manual orienting responses made in both the Tactile Stimulation (Panel B1) and No Stimulation Baseline (Panel B3) groups. Manual orientations that were contralateral and ipsilateral to the cerebral hemisphere receiving the tactile stimulus (in the Tactile Stimulation group) or the nearest of the two auditory stimuli (in the No Stimulation Baseline group) are scored separately. Correct 
localizations in the Tactile Stimulation group are represented by contralateral responses in the uncrossed-hands posture, and ipsilateral responses in the crossed-hands posture. We predicted there to be no robust directional trends in the No Stimulation Baseline group (in either posture). Responses toward the closest activated buzzer would be contralateral in direction, regardless of posture.

In order to compare infants' manual orienting to tactile stimuli against baseline performance, we calculated a proportion score (proportion of directional manual responses that were contralateral) for each posture. The proportion of manual contralateral responses was significantly higher in the Tactile Stimulation group than in the No Stimulation Baseline group in the Uncrossed-hands posture $(\underline{\mathrm{t}}(22)=3.6, \underline{\mathrm{p}}<.003, \underline{\mathrm{d}}=1.4)$, but not the Crossed-hands condition $(\underline{\mathrm{t}}(22)=1.0$, $\underline{\mathrm{n} . \mathrm{s}}$. $\underline{\mathrm{d}}=.4)$.

A 2 x 2 repeated-measures ANOVA (Posture: Uncrossed-hands / Crossed-hands; Response direction: Contralateral / Ipsilateral) on the number of manual responses in the Tactile Stimulation group revealed a significant main effect of Response direction $\left(\underline{\mathrm{F}}(1,12)=6.5, \underline{\mathrm{p}}<.03, \underline{\mathrm{\eta}}_{p}=.35\right)$ indicating that these younger infants tended to make contralateral manual responses which were appropriate to the uncrossed-hands posture, across both the uncrossed-hands and crossed-hands postures. There was also a significant interaction between Posture and Response direction $\left(\underline{\mathrm{F}}(1,12)=14.4, \underline{\mathrm{p}}<.004, \underline{\eta_{p}}=.25\right)$, demonstrating that the infants were able to modulate their manual orienting responses following a change of posture. The main effect of posture was not significant $\left(\underline{\mathrm{F}}(1,12)=2.0, \underline{\underline{\eta}}_{p}^{2}=.15\right)$. Planned comparisons of responses toward the ipsilateral and contralateral sides within the two postures indicated significantly more manual responses were made to the contralateral side of space in the Uncrossed-hands posture $(\underline{t}(12)=4.6$, one-tailed $\underline{\mathrm{p}}<.001, \underline{\mathrm{d}}=1.3)$. No significant difference was observed in the Crossed-hands posture $(\underline{\mathrm{t}}<1.5)$. 
Visual orienting. Figure 3B displays the visual orienting responses made in both the Tactile Stimulation (Panel B2) and No Stimulation Baseline (Panel B4) groups. Overt visual orienting responses that were contralateral and ipsilateral to the hemisphere receiving the tactile stimulus (in the Tactile Stimulation group) or the nearest of the two auditory stimuli (in the No Stimulation Baseline group) were scored separately. As in Experiment 1, correct localizations in the Tactile Stimulation group are represented by contralateral responses in the uncrossed-hands posture, and by ipsilateral responses in the crossed-hands posture. We did not predict any robust directional trends in the No Stimulation Baseline group (in either posture). Responses toward the closest activated buzzer would be contralateral in direction, regardless of posture.

In order to compare infants' visual orienting to tactile stimuli against baseline performance, we calculated a proportion score (proportion of directional responses that were contralateral) for each posture. The performance of the Tactile Stimulation and No Stimulation Baseline groups was also compared using planned comparisons. The proportion of contralateral visual responses was significantly higher in the Tactile Stimulation group, than in the No Stimulation Baseline group in the Uncrossed-hands posture $(\underline{\mathrm{t}}(17)=2.7, \underline{\mathrm{p}}<.014, \underline{\mathrm{d}}=1.3)$, but not the Crossed-hands posture $(\underline{\mathrm{t}}(12)=1.2$, $\underline{\mathrm{n} . \mathrm{s}}$, $\underline{\mathrm{d}}=0.8)$.

A $2 \times 2$ repeated-measures ANOVA was also conducted on the number of visual responses in the Tactile Stimulation group (Posture: Uncrossed-hands / Crossed-hands; Response direction: Contralateral / Ipsilateral). Despite numerical trends indicating fewer responses overall in the crossed-hands posture, and also fewer ipsilateral than contralateral responses in the uncrossed-hands posture, no main effects or interactions reached significance. Nonetheless, planned comparisons of responses toward the ipsilateral and contralateral sides within the two posture conditions indicated that the 
infants made significantly more contralateral than ipsilateral visual directional responses in the Uncrossed-hands posture $(\underline{\mathrm{t}}(12)=2.1$, one-tailed $\mathrm{p}<.03, \underline{\mathrm{d}}=.63)$.

\section{Discussion}

The results of Experiment 2 extend the findings of Experiment 1 to a younger age group. The 6.5-month-olds tested in the present study responded both manually and visually to the unseen tactile stimuli presented to their palms. However, in contrast to the 10-month-olds tested in Experiment 1, the younger infants tested in this study made substantially fewer and later visual than manual responses to the tactile stimuli, across the same number of trials (10).

Despite a lower level of visual orienting to tactile stimuli than that shown by the 10-month-olds, the 6.5-month-old infants tested here were nevertheless still able to orient their eyes toward tactile stimuli presented to their hands in the familiar uncrossed-hands posture; significantly more manual and visual localizations were made toward the hand that had received vibrotactile stimulation than toward the unstimulated hand (see Figure 3, Panels B1 and B2). The reduced number of visual responses in this age group, particularly in the crossed-hands posture, makes it difficult to address the question of whether or not the infants were able to update their visual orienting responses in order to take account of the new posture of their limbs. Reduced responding in the crossed-hands posture may have been due to either this age group's uncertainty concerning the location of the stimulus in this posture, or to a suppression of their responses as a result of the unfamiliar posture being adopted. Nonetheless, both interpretations point to much less robust visual orienting to tactile stimuli at 6.5 months of age.

Manual responses, however, were relatively plentiful in both postures (see Figure 3, Panel B1). Despite the appropriate directional trends in the manual orienting responses in each posture, a main effect of response direction indicated that, in general, the 6.5- 
month-olds made more contralateral than ipsilateral responses (and indeed the trend to respond appropriately did not reach significance in the crossed-hands posture). The contralateral response is correct in the more familiar uncrossed-hands posture, but inappropriate for the crossed-hands posture. Manual responses to the (visual) contralateral location across both postures indicate that there is an influence of a visual code on this age group's manual orienting responses, even though manual orienting could, in principle, simply rely on a body-centred frame of reference (cf. Kitazawa, 2002; Soto-Faraco, Ronald, \& Spence, 2004).

Both manual and visual responses in the Tactile Stimulation condition were made slightly later than those made in the No Stimulation Baseline condition in which no tactile stimulus was presented (see Figure 2B). In the absence of any task-relevant somatosensory stimulation in the No Stimulation Baseline condition, it seems likely that the responses were driven by visual stimuli. Thus, we may explain the reduced speed of orienting in the Tactile Stimulation condition as due to less efficient spatial coding of somatosensory than visual locations. A similar argument has been advanced by Groh and Sparks (1996a) who found that saccades to somatosensory targets take noticeably longer than those directed toward visual targets. In addition, it is also possible that the delay was due to a certain amount of surprise concerning the nature of the stimuli. It seems likely that much of the tactile stimulation that 6.5 -month-old infants experience would normally be synchronous with some visual change in the environment (at least for tactile stimuli presented on their hands). Thus, it may also be that the delay represents a suppression of responses in the Tactile Stimulation condition due to uncertainty concerning the location of the tactile stimuli, or to an expectation for the appearance of correlated visual stimuli. 
Previous research into the early development of peripersonal spatial awareness has focussed on the development of crossmodal links between vision and proprioceptive control (von Hofsten, 1979, 1982; von Hofsten \& Fazel-Zandy, 1984; von Hofsten \& Rönnqvist, 1988). The experiments reported in the present study are the first to investigate infants' peripersonal spatial perception by examining their localization of (invisible) tactile stimuli. We observed two age groups' (6.5-month-olds and 10-month-olds) manual and visual orienting responses to vibrotactile stimuli presented to the palms of their hands in either uncrossed or crossed-hands postures.

\section{Tactile localization and crossmodal orienting to touch}

Infants in both age groups were able to localize/orient toward (invisible) tactile stimuli correctly in the uncrossed-hands posture: More manual and visual responses were directed toward the stimulated rather than to the unstimulated hand (see Figure 3). The ability to direct responses to tactile stimuli in the absence of any visual spatial cue suggests that both age groups can make use of purely unimodal tactile information in order to explore and act upon their peripersonal environment. However, in order to determine whether the sensorimotor behaviors observed here represent cortically mediated, prospective action (e.g., von Hofsten, 2004, 2007), it is important to consider the kinds of neural circuitry that underlies spatial performance. A number of neonatal cutaneous responses demonstrate spatial directionality dependent on the particular site of stimulation. These "transient reflexes" have traditionally been thought of as being governed by spinal sensorimotor loops (though see von Hofsten, 2004, for an interpretation of some of these behaviors in terms of prospective control). In both of the age groups tested here, the majority of visual and manual responses measured emerged between 1 and 4 seconds after stimulus presentation (see Figure 2). The latency of these responses argues against a spinal reflex, as the muscular activity associated with 
cutaneous spinal reflex responses is typically observed at a latency of around $20 \mathrm{~ms}$, even in the newborn infant (see Issler \& Stephens, 1983). Another indication that these responses were under cortical control comes from the comparison of the speed of 6.5month-olds' responses against that of a baseline group who were not presented with any vibrotactile stimulation (Experiment 2). The latency of infants' responses to the tactile stimuli was greater than that measured in the No Stimulation Baseline group (see Figure 2B), suggesting that the infants' orienting responses to tactile-only stimuli followed more complicated neural pathways than those implicated in spinal reflex loops.

Despite the accurate tactile localization observed in both age groups, the crossmodal overt orienting of visual attention toward tactile stimulation appears to increase in frequency and speed between 6.5 and 10 months of age such that visual latencies, significantly delayed relative to manual orienting responses at 6.5 months, emerge at the same latency as manual responses (if not slightly before) by 10 months of age. It is thus unclear whether the younger age group's visual orienting responses are made in response to the tactile stimuli or in response to the visual cues produced by their own manual responses ${ }^{4}$. Nevertheless, the increase in directional visual orienting responses from 6.5 to 10 months of age suggests a development of cross-modal links in overt attentional response to the location of tactile stimuli.

Spatial remapping of visual responses across change in posture

We tested 6.5- and 10-month-old infants' ability to localize vibrotactile stimuli in two different hand postures: An uncrossed-hands posture and a less familiar crossedhands posture (see Figure 1). The relation of tactile locations to the visual framework changes with this postural manipulation, and thus, in order to locate a tactile stimulus visually, a spatial remapping between tactile and visual space is required (cf. Jay \& Sparks, 1984; Pöppel; 1973; Spence, McDonald, \& Driver, 2004). Adoption of the 
crossed-hands posture alters the familiar mapping of tactile to visual location such that the tactile location that would normally project to the same cerebral hemisphere as the visual representation of the hand, now arrives at the opposite hemisphere (see Lloyd et al., 2003; Spence, Pavani, \& Driver, 2000). In the uncrossed-hands posture, the appropriate visual orienting response was toward the side of space contralateral to the hemisphere receiving the somatosensory input associated with the tactile stimulus. In the crossed-hands posture, the infants would have had to remap visual orienting responses toward the ipsilateral visual field instead. Our study is thus the first to examine the development of crossmodal spatial remapping contingent upon postural change in infants.

In the 10-month-olds (tested in Experiment 1), we observed an interaction between the direction of visual responses and the posture in which the hands were placed (see Figure 3, Panel A2). The numerical trends indicated large, but non-significant, appropriate directional trends in each posture condition, suggesting that the 10 -montholds were able to modulate their visual orienting behaviors with respect to changes in the posture of their hands. At 6.5 -months of age, the small number of visual orientations observed limited our ability to assess any remapping of visual responses (see Figure 3, Panel B2). However, this younger age group demonstrated significantly more contralateral responses in the uncrossed-hands posture, and a relatively even number of contralateral and ipsilateral visual responses in the crossed-hands posture. Thus, it appears that some degree of visual-tactile integration was taking take place at 6.5-months, but that appropriate visual orienting in unfamiliar postures emerges between 6.5 and 10 months.

\section{Influence of a visual code on tactile spatial perception}

Both age groups demonstrated an ability to make accurate manual responses to the tactile stimuli (see Figure 3, Panels A1 and B1). In the less familiar, crossed-hands posture, the 10-month-olds also showed significantly more responses to the stimulated 
hand, whereas the 6.5-month-olds demonstrated a trend to respond toward the stimulated hand. On first consideration, the ability to make appropriate manual responses across postural change is not that surprising. Computationally speaking, no reference to a visual frame of reference is required as the manual localization of tactile stimuli can, at least in theory, be made by reference to a tactile (body-centred) spatial map alone, regardless of limb position. However, despite the preponderance of accurate manual localizations in both postures, the younger age group (6.5-month-olds) showed a significant overall bias to respond manually toward the visual location contralateral to the cerebral hemisphere receiving somatosensory input from the hand. Accordingly, they made a smaller proportion of correct manual responses in the crossed-hands posture (see Figure 3, Panel B1). The latency analysis indicates that manual responses emerged earlier than visual responses in this age group (see Figure 2B). It therefore seems that, despite the adequacy of the body-centred frame of reference in the absence of visual orienting behavior, a visual framework is used spontaneously to coordinate manual orientation responses (cf. Kitazawa, 2002; Soto-Faraco et al., 2004, for consistent evidence that adult humans cannot help but refer to a more external, possibly visual, representation when making purely tactile discrimination responses). The influence of a visual spatial framework on 6.5-month-olds' manual responses strongly suggests that this age group perceives tactile sensations with respect to visual spatial coordinates.

This finding is in line with recent research demonstrating that, in typical adult participants, tactile sensations are recruited into a visual spatial framework such that posture affects the accuracy of TOJs; with poorer performance being observed in the crossed-hands posture (Azañón \& Soto-Faraco, submitted; Kóbor et al., 2006; Röder et al., 2004; Schicke \& Röder, 2006; Shore et al., 2002; Yamamoto \& Kitazawa, 2001) as compared to the uncrossed-hands posture. On the basis of these similar postural effects 
we might therefore conclude that there is continuity in the use of a visual code for tactile localization between 6.5 months of age and adulthood. However, if this is the case, we must also consider why the 10-month-olds tested in Experiment 1 showed no contralateral bias in their manual responding (see Figure 3, Panel A1).

An important difference between the paradigm used here with infants and those typically used with adults (Azañón \& Soto-Faraco, submitted; Kóbor et al., 2006; Röder et al., 2004; Shore et al., 2002; Yamamoto \& Kitazawa, 2001) is that the adult participants were required to make decisions about the temporal order of two stimuli (one presented to each hand). In a task requiring adults to make a saccade to a single tactile stimulus applied to the hand, Groh and Sparks (1996a) demonstrated that saccades were made in the correct direction, but with markedly curved trajectories when the hands were positioned in a crossed-hands posture. However, when a delay of 600-1000 ms was introduced between the target presentation and saccadic response, straight (i.e., accurate) saccades were observed in both postures. The great majority of the visual and manual responses made by the 10-month-olds occurred at least $1000 \mathrm{~ms}$ after the presentation of the tactile stimulus. Thus, it may be that a tactile stimulus on one hand alone, as is used in the current study (and in Groh \& Sparks, 1996a, study), is simple enough for 10-month-olds (and adults) to be able to track across the midline, even when using a primarily visual spatial code for their manual responses (for convergent psychophysical evidence in favour of this interpretation see Holmes, Sanabria, Calvert, \& Spence; 2006; Spence, Baddeley, Zampini, James, \& Shore, 2003). Use of a single visual frame of reference by the 10month-olds is also suggested by the close temporal relationship between their visual and manual responding.

Thus, although adults use a visual frame of reference in locating (and responding to) somatosensory stimuli, simpler kinds of spatial localization appear to take account of 
postural changes across the midline. It seems likely therefore that the single tactile stimulus used in the experiments reported in the present study may be simple enough for 10-month-olds, but not 6.5-month-olds, to track in relation to a visual frame of reference even across changes in limb position.

Developmental change in multisensory interactions in the body schema and peripersonal space

The findings reported here indicate that infants appear to utilize a visual frame of reference to locate tactile stimulation by 6.5 months of age. We also argue that improvements between 6.5 and 10 months of age in manual and visual localization performance in the unfamiliar crossed-hands posture reflect increasing competence at remapping the location of tactile stimuli in visual space across changes in limb position. Visually guided reaching and grasping behaviors have been observed to emerge at around 4 to 5 months of age (Thelen, Corbetta, Kamm, Spencer, Schneider, \& Zernicke, 1993; von Hofsten, 1984), with developments in the planned nature of this behavior continuing into the second half of the first year (von Hofsten \& Fazel-Zandy, 1984; von Hofsten \& Ronnqvist, 1988). Infants' ability to reach to and across the midline for visually presented objects whilst the ipsilateral arms are restrained emerges between 4 and 6 months of age (see Morange \& Bloch, 1996; Provine \& Westerman, 1979). Spontaneous midline crossing also appears to emerge at about 6 months of age, in the context of having to use both hands to lift large or heavy objects (van Hof, van der Kamp, \& Savelsbergh, 2002). It would therefore seem likely therefore that the emergence of reaching across the midline provides infants with the necessary multisensory experience to update representations of the location of their limbs (the "body schema"; Holmes \& Spence, 2006; Maravita, Spence, \& Driver, 2004), and thus the location of tactile stimuli in visual peripersonal space. 
One question that remains to be answered concerns when touch is initially recruited into a visual spatial framework. Research comparing tactile localization in congenitally blind, late blind, and sighted adult participants (Röder et al., 2004) indicates that postural effects on TOJs emerge only when the participant has had visual experience in the first years of life. The 6.5-month-olds tested here showed postural effects on their manual localization, indicating their use of a visual frame of reference even at this early stage of development. It is certainly possible that the initial recruitment of touch by the visual frame occurs earlier in development. This question can be resolved by the development of the current paradigm in order to compare postural effects in age groups across the first six months of life.

Despite the early influence of vision on tactile perception reported in the present study, it is also possible that the development of multisensory integration responsible for the differences between postural effects on TOJ accuracy in congenitally blind and sighted participants (Röder et al., 2004) occurs later in infancy or during childhood. There are some indications that the relative roles of vision and proprioception in directing action undergo development during childhood. For example, Renshaw (1930) showed many years ago that when blindfolded, children are much better at orienting proprioceptively to tactile stimuli than are adults; suggesting that adults rely much more on vision for directing proprioceptive responses within peripersonal space (see also Warren \& Pick, 1970). The natural correlation of proprioceptive and tactile sensations within the bodycentred frame of reference makes it likely that touch should follow a similar developmental trajectory to proprioception in terms of its integration with vision.

$\underline{\text { Summary }}$

6.5- and 10-month-old infants used tactile stimuli in isolation as a cue to respond to their peripersonal spatial environment. At 6.5 months of age, the infants' responses are 
most evident in proprioceptive manual control, but by 10 months of age visual orienting behavior becomes more important. An ability to remap visual and manual responses to tactile stimuli across changes in the posture of the hands undergoes development between 6.5 and 10 months. The younger age group made appropriate manual responses in the uncrossed-hands posture, but in the unfamiliar crossed-hands posture showed a relatively even number of appropriate and inappropriate orienting responses. In the crossed-hands posture, the production of manual responses which would have been directionally appropriate in the more familiar uncrossed-hands posture of the arms indicates the influence of a visual spatial framework on proprioceptive manual control. We argue that this is due to an early developing cross-modal interaction in which a visual spatial frame of reference is used to localize tactile stimuli. Furthermore, we suggest that developments in the ability to remap visual and manual directional responses across changes in posture may be due to the development of a more accurate body schema driven by the increased experience of crossing the midline which infants gain after 6 months of age. 


\section{References}

Abravanel, E. (1981). Integrating the information from eyes and hands: A developmental account. In R. D.Walk \& H. L. Pick, Jr. (Eds.), Intersensory perception and sensory integration (pp.71-108). New York: Plenum.

Acredolo, L. P. (1978). Development of spatial orientation in infancy. Developmental Psychology, 14, 224-234.

Aronson, E., \& Rosenbloom, S. (1971). Space perception in early infancy: Perception with a common auditory-visual space. Science, 172, 1161-1163.

Azañón, E., \& Soto-Faraco, S. (submitted). Alleviating the 'crossed-hands' deficit by seeing uncrossed rubber hands. Experimental Brain Research.

Bremner, A. J., Bryant, P. E., \& Mareschal, D. (2006). Object-centred spatial reference in 4-month-old infants. Infant Behavior and Development, 29, 1-10.

Bremner, J. G. (1978). Egocentric versus allocentric spatial coding in 9-month-old infants: Factors influencing the choice of code. Developmental Psychology, 14, 346-355.

Bremner, J. G., Johnson, S. P., Slater, A. M., Mason, U., \& Spring, J. (2006). Crossmodal perception of object trajectories in infancy. Paper presented as part of the symposium, "Developing crossmodal representations of objects and space". International Conference on Infancy Studies, Kyoto, Japan, June 2006.

Bryant, P.E., Jones, P., Claxton, V., \& Perkins, G.M. (1972). Recognition of shapes across modalities by infants. Nature, 240, 303-304.

Butterworth, G., \& Castillo, M. (1976). Coordination of auditory and visual space in newborn human infants. Perception, 5, 155-160. 
Clifton, R. K., Morrongiello, B. A., Kulig, J., \& Dowd, J. (1981). Newborns' orientation toward sound: Possible implications for cortical development. Child Development, 52, 833-838.

Eimer, M. (2001). Crossmodal links in spatial attention between vision, audition, and touch: Evidence from event-related brain potentials. Neuropsychologia, 39, 1292-1303.

Eimer, M. (2004). Multisensory integration: How visual experience shapes spatial perception. Current Biology, 14, R115-R117.

Fényes, I., Gergely, C., \& Tóth, S. (1960). Clinical and electromyographic studies of "spinal reflexes" in premature and full-term infants. Journal of Neurology, Neurosurgery \& Psychiatry, 23, 63-68.

Flanery, R. C., \& Balling, J. D. (1979). Developmental changes in hemispheric specialization for tactile spatial ability. Developmental Psychology, 15, 364372.

Ghent, L. (1961). Developmental change in tactual thresholds on dominant and nondominant sides. Journal of Comparative and Physiological Psychology, 54, 670-673.

Gilmore, R. O., \& Johnson, M. H., (1997). Body-centered representations for visually-guided action emerge in early infancy. Cognition, 65, B1-B9.

Graziano, M. S. A., Cooke, D. F., \& Taylor, C. S. R. (2000). Coding the location of the arm by sight. Science, 290, 1782-1786.

Graziano, M. S. A., \& Gross, C. G. (1993). A bimodal map of space: Somatosensory receptive fields in the macaque putamen with corresponding visual receptive fields. Experimental Brain Research, 97, 96-109. 
Graziano, M. S. A., \& Gross, C. G. (1994). Mapping space with neurons. Current Directions in Psychological Science, 3, 164-167.

Graziano, M. S. A., Gross, C. G., Taylor, C. S. R., \& Moore, T. (2004). A system of multimodal areas in the primate brain. In C. Spence \& J. Driver (Eds.), Crossmodal space and crossmodal attention (pp. 51-67). Oxford: Oxford University Press.

Graziano, M. S. A., Yap, G. S., \& Gross, C. G. (1994). Coding of visual space by premotor neurons. Science, 266, 1054-1057.

Gregory, R. L. (1963). Distortion of visual space as inappropriate constancy scaling. Nature, 199, 678-691.

Groh, J. M., \& Sparks, D. L. (1996a). Saccades to somatosensory targets: 1. Behavioral characteristics. Journal of Neurophysiology, 75, 412-427.

Groh, J. M., \& Sparks, D. L. (1996b). Saccades to somatosensory targets: 2. Motor convergence in primate superior colliculus. Journal of Neurophysiology, 75, 428-438.

Groh, J. M., \& Sparks, D. L. (1996c). Saccades to somatosensory targets: 3. Eyeposition dependent somatosensory activity in primate superior colliculus. Journal of Neurophysiology, 75, 439-453.

Hatta, T., Yamamoto, M., Kawabata, Y., \& Tsutui, K. (1981). Development of hemisphere specialization for tactile recognition in normal children, Cortex, 17, 611-616.

Holmes, N. P., Sanabria, D., Calvert, G. A., \& Spence, C. (2006). Multisensory interactions follow the hands across the midline: Evidence from a non-spatial visual-tactile congruency task. Brain Research, 1007, 108-115. 
Holmes, N. P., \& Spence, C. (2006). Beyond the body schema: Visual, prosthetic, and technological contributions to bodily perception and awareness. In G. Knoblich, I. M. Thornton, M. Grosjean, \& M. Shiffrar (Eds.), Human body perception from the inside out (pp. 15-64). Oxford: Oxford University Press.

Iriki, A., Tanaka, M., \& Iwamura, Y. (1996). Coding of modified body schema during tool use by macaque postcentral neurones. Neuroreport, 7, 2325-2330

Issler, H., \& Stephens, J. A. (1983). The maturation of cutaneous reflexes studied in the upper limb in man. Journal of Physiology, 335, 643-654.

Jay, M. F., \& Sparks, D. L. (1984). Auditory receptive fields in primate superior colliculus shift with changes in eye position. Nature, 309, 345-347.

Kaufman, J., \& Needham, A. (1999). Objective spatial coding by 6.5 -month-old infants in a visual dishabituation task. Developmental Science, 2, 432-441.

Kennett, S., Spence, C., \& Driver, J. (2002). Visuo-tactile links in covert exogenous spatial attention remap across changes in unseen hand posture. Perception \& Psychophysics, 64, 1083-1094.

Kisilevsky, B. S., \& Muir, D. W. (1984). Neonatal habituation and dishabituation to tactile stimulation during sleep. Developmental Psychology, 20, 367-373.

Kitazawa, S. (2002). Where conscious sensation takes place. Consciousness and Cognition, 11, 475-477.

Kóbor, I., Füredi, L., Kovács, G., Spence, C., \& Vidnyánszky, Z. (2006). Back-tofront: Improved tactile discrimination performance in the space you can't see. Neuroscience Letters, 400, 163-167. 
Ladavas, E., \& Farnè, A. (2004). Neuropsychological evidence for multimodal representations of space near specific body parts. In C. Spence \& J. Driver (Eds.), Crossmodal space and crossmodal attention (pp. 51-67). Oxford: Oxford University Press.

Lloyd, D. M., Shore, D. I., Spence, C., \& Calvert, G. A. (2003). Multisensory representation of limb position in human premotor cortex. Nature Neuroscience, 6, 17-18.

Maravita, A., Spence, C., \& Driver, J. (2003). Multisensory integration and the body schema: Close to hand and within reach. Current Biology, 13, R531-R539.

Mareschal, D., \& Johnson, M. H. (2003). The "what" and "where" of infant object representations. Cognition, 88, 259-276.

McGraw, M. B. (1943). The neuromuscular maturation of the human infant. New York: Columbia University Press.

Molina, M., \& Jouen, F. (1998). Modulation of the palmar grasp behaviour in neonates according to texture property. Infant Behavior and Development, 21, 659-666.

Molina, M., \& Jouen, F. (2001). Modulation of manual activity by vision in human newborns. Developmental Psychobiology, 38, 123-132.

Morange, F., \& Bloch, H. (1996). Lateralization of the approach movement and the prehension movement in infants from 4 to 7 months. Early Development and Parenting, 5, 81-92.

Moreau, T., Helfgott, E., Weinstein, P., \& Milner, P. (1978). Lateral differences in habituation of ipsilateral head-turning to repeated tactile stimulation in the human newborn. Perceptual and Motor Skills, 46, 427-436.

Moreau, T., \& Milner, P. (1981). Lateral differences in the detection of touched body parts in young children. Developmental Psychology, 17, 351-356. 
Morrongiello, B. A., Fenwick, K. D., \& Chance, G. (1998). Crossmodal learning in newborn infants: Inferences about properties of auditory-visual events. Infant Behavior \& Development, 21, 543-554.

Muir, D. W., \& Field, J. (1979). Newborn infants orient to sounds. Child Development, 50, 431-436.

Neil, P. A., Chee-Ruiter, C., Scheier, C., Lewkowicz, D. J., \& Shimojo, S. (2006). Development of multisensory spatial integration and perception in humans. Developmental Science, 9, 454-464.

Newcombe, N. S., Sluzenski, J., \& Huttenlocher, J. (2005). Pre-existing knowledge versus on-line learning: What do young infants really know about spatial location? Psychological Science, 16, 222-227.

Nissen, H. W., Chow, K. L., \& Semmes, J. (1951). Effects of restricted opportunity for tactual, kinesthetic, and manipulative experience on the behavior of a chimpanzee. American Journal of Psychology, 64, 485-507.

Piaget, J. (1952). The origins of intelligence in the child. London: Routledge \& Kegan-Paul.

Pöppel, E. (1973). Comments on "Visual system's view of acoustic space". Nature, 243, 231

Provine, R. R., \& Westerman, J. A. (1979). Crossing the midline: Limits of early eyehand behavior. Child Development, 50, 437-441.

Quinn, P. C. (1994). The categorization of above and below spatial relations by young infants. Child Development, 65, 58-69.

Renshaw, S. (1930). The errors of cutaneous localization and the effect of practice on the localizing movement in children and adults. Journal of Genetic Psychology, 38, 223-238. 
Rizzolatti, G., Fadiga, L., Fogassi, L., \& Gallese, V. (1997). The space around us. Science, 277, 190-191.

Röder, B., Rösler, F., \& Spence, C. (2004). Early vision impairs tactile perception in the blind. Current Biology, 14, 121-124.

Sann, C., \& Streri, A. (2007). Perception of object shape and texture in human newborns: Evidence from cross-modal transfer tasks. Developmental Science, 10, 399-410.

Schicke, T., \& Röder, B. (2006). Spatial remapping of touch: Confusion of perceived stimulus order across hand and foot. Proceedings of the National Academy of Sciences, 103, 11808-11813.

Sherrington, C. S. (1910). Flexion-reflex of the limb, crossed extension-reflex, and reflex stepping and standing. Journal of Physiology, 40, 28-121.

Shore, D. I., Spry, E., \& Spence, C. (2002). Confusing the mind by crossing the hands. Cognitive Brain Research, 14, 153-163.

Soto-Faraco, S., Ronald, A., \& Spence, C. (2004). Tactile selective attention and body posture: Assessing the contribution of vision and proprioception. Perception \& Psychophysics, 66, 1077-1094.

Spelke, E. (1976). Infants' intermodal perception of events. Cognitive Psychology, 8, 553-560.

Spence, C., Baddeley, R., Zampini, M., James, R., \& Shore, D. I. (2003). Crossmodal temporal order judgments: When two locations are better than one. Perception \& Psychophysics, 65, 318-328.

Spence, C., McDonald, J., \& Driver, J. (2004). Exogenous spatial-cuing studies of human crossmodal attention and multisensory integration. In C. Spence \& J. Driver (Eds.), Crossmodal space and crossmodal attention (pp. 277-320). Oxford: Oxford University Press. 
Spence, C., Pavani, F., \& Driver, J. (2000). Crossmodal links between vision and touch in covert endogenous spatial attention. Journal of Experimental Psychology: Human Perception \& Performance, 26, 1298-1319.

Spence, C., Pavani, F., \& Driver, J. (2004). Spatial constraints on visual-tactile crossmodal distractor congruency effects. Cognitive, Affective, \& Behavioral Neuroscience, 4, 148-169.

Spence, C., Pavani, F., Maravita, A., \& Holmes, N. (2004). Multisensory contributions to the 3-D representation of visuotactile peripersonal space in humans: Evidence from the crossmodal congruency task. Journal of Physiology (Paris), 98, 171-189.

Spence, C., \& Walton, M. (2005). On the inability to ignore touch when responding to vision in the crossmodal congruency task. Acta Psychologica, 118, 47-70.

Streri, A., \& Gentaz, E. (2003). Cross-modal recognition of shape from hand to eyes in human newborns. Somatosensory and Motor Research, 20, 13-18.

Streri, A., \& Gentaz, E. (2004). Cross-modal recognition of shape from hand to eyes and handedness in human newborns. Neuropsychologia, 42, 1365-1369.

Tan, U., \& Tan, M. (1999). Incidences of asymmetries for the palmar grasp reflex in neonates and hand preference in adults. Neuroreport, 10, 3253-3256.

Thelen, E., Corbetta, D., Kamm, K., Spencer, J. P., Schneider, K., \& Zernicke, R. F. (1993). The transition to reaching: Mapping intention and intrinsic dynamics: Developmental biodynamics: Brain body, behavior connections. Child Development, 64, 1058-1098.

Van Hof, P., van der Kamp, J., \& Savelsbergh, G. J. P. (2002). The relation of unimanual and bimanual reaching to crossing the midline. Child Development, $\mathbf{7 3}, 1353$ 1362. 
Von Hofsten, C. (1979). Development of visually guided reaching: The approach phase. Journal of Human Movement Studies, 5, 160-178.

Von Hofsten, C. (1982). Eye-hand coordination in the newborn. Developmental Psychology, 18, 450-461.

Von Hofsten, C. (1984). Developmental changes in the organization of prereaching movements. Developmental Psychology, 20, 378-388.

Von Hofsten, C. (2004). An action perspective on motor development. Trends in Cognitive Sciences, 8, 266-272.

Von Hofsten, C. (2007). Action in development. Developmental Science, 10, 54-60.

Von Hofsten, C., \& Fazel-Zandy, S. (1984). Development of visually guided hand orientation in reaching. Journal of Experimental Child Psychology, 38, 208-219.

Von Hofsten, C., \& Rönnqvist, L. (1988). Preparation for grasping an object: A developmental study. Journal of Experimental Psychology: Human Perception and Performance, 14, 610-621.

Warren, D. H., \& Pick, H. L. (1970). Intermodality relations in blind and sighted people. Perception \& Psychophysics, 8, 430-432.

Wertheimer, M. (1961). Psychomotor coordination of auditory and visual space at birth. Science, 134, 1692.

Yamamoto, S., \& Kitazawa, S. (2001). Reversal of subjective temporal order due to arm crossing. Nature Neuroscience, 4, 759-765.

Zappella, M., \& Simopoulos, A. (1966). The crossed-extension reflex in the newborn. Annales Paediatriae Fenniae, 12, 30-33. 


\section{Table Captions}

Table 1: $\quad$ Mean number of trials (out of 10) on which infants made i) No response ("None"), ii) A manual response only ("Manual only"), iii) A visual response only ("Visual only"), or iv) A manual and a visual response ("Both"). Infants are grouped by age group (10-month-olds (Experiment 1) and 6.5-month-olds (Experiment 2)). Figures in brackets indicate the standard deviations of the means. 
Figure Captions

Figure 1: A 6.5-month-old infant shown adopting the uncrossed- and crossed-hands postures (Panels A \& B, respectively). A 6.5-month-old is displayed here in order to facilitate visual comparison of the postures (in Experiment 2, 6.5-month-olds were tested in both uncrossed- and crossed-hands postures).

Figure 2: Latency of visual and manual responses following trial onset. The line graphs display the cumulative number of first responses across all trials plotted as a function of response latency. The inset bar graphs display the mean latencies of the infants' first responses. Panel A displays cumulative responses and mean latencies of visual and manual responses of the 10month-olds tested in Experiment 1. Panel B displays cumulative responses and mean latencies of visual and manual responses of the 6.5-month-olds tested in Experiment 2, with 'Tactile Stimulation' (TS) and 'No Stimulation Baseline' (NSB) groups plotted separately. The error bars represent the SE of the means, and asterisks indicate statistically significant comparisons $(* *=\underline{p}<.01)$.

Figure 3: Number of trials in which the first directional visual and manual responses were toward the contralateral and ipsilateral visual fields in the uncrossedhands and crossed-hands postures. Ten-month-olds' (Exp. 1) responses (out of 10 trials) are displayed in Panels A1 (manual) and A2 (visual). Posture is compared between infants in Experiment 1. Six-and-a-halfmonth-olds' (Exp. 2) responses (out of 5) are displayed in Panels B1-B4. (Panel B1 = Manual responses in the Tactile Stimulation group; Panel B2 = 
Visual responses in the Tactile Stimulation group; Panel B3 = Manual responses in the No Stimulation Baseline group; Panel B4 = Visual responses in the No Stimulation Baseline group). The error bars represent the SE of the means, and the asterisks indicate statistically significant comparisons $(*=\underline{p}<.05, * * *=\underline{p}<.001)^{3}$. 


\section{Footnotes}

1. Initially, we had planned to compare performance within individual infants by presenting two blocks of 10 trials each, with the order counterbalanced, but the rapid habituation of responses to the tactile stimuli observed during the first block of trials dictated that we examine the effect of posture between groups instead.

2. Four infants did not provide mean visual response latency scores in the No Stimulation Baseline condition, as they made no visual responses during their testing session.

3. The visual data in the No Stimulation Baseline condition of Experiment 2 was markedly non-normal, due to low levels of visual responses. Though a significant preference for ipsilateral responding was observed in the crossed-hands posture using a paired samples t-test, a non-parametric binomial sign test failed to uncover a significant difference $(\mathrm{p}=.125)$. Because we can see no likely reason why infants would respond more ipsilaterally in this condition (away from the closest auditory stimulus), we consider the results of the t-test to be (most likely) spurious.

4. Indeed, the development of crossmodal orienting of vision to touch may likely be bootstrapped by the detection of manual responses. Future research might clarify the developmental relations between visual and manual orienting to touch by investigating whether different age groups will visually orient toward touches applied to both seen and unseen hands (cf. Groh \& Sparks, 1996a). 
5. We judged that the arm-crossing paradigm used in the experiments reported here would have been difficult to conduct with pre-reaching infants, due to the shorter length of their arms. 
Table 1

$\begin{array}{ccc}\text { Manual } & \begin{array}{c}\text { Visual } \\ \text { only }\end{array} & \text { Both }\end{array}$

\section{Experiment 1:}

10-month-olds

$(\mathrm{N}=12)$

$3.17(2.25) \quad 2.58(1.93) \quad 2.42(1.68) \quad 1.83(1.64)$

Experiment 2:

6.5-month-olds

$(\mathrm{N}=24)$

$2.54(1.74) \quad 4.38(2.06) \quad 0.33(0.64) \quad 2.75(1.54)$ 
Figure 1
(A) Uncrossed-hands posture
(B) Crossed-hands posture

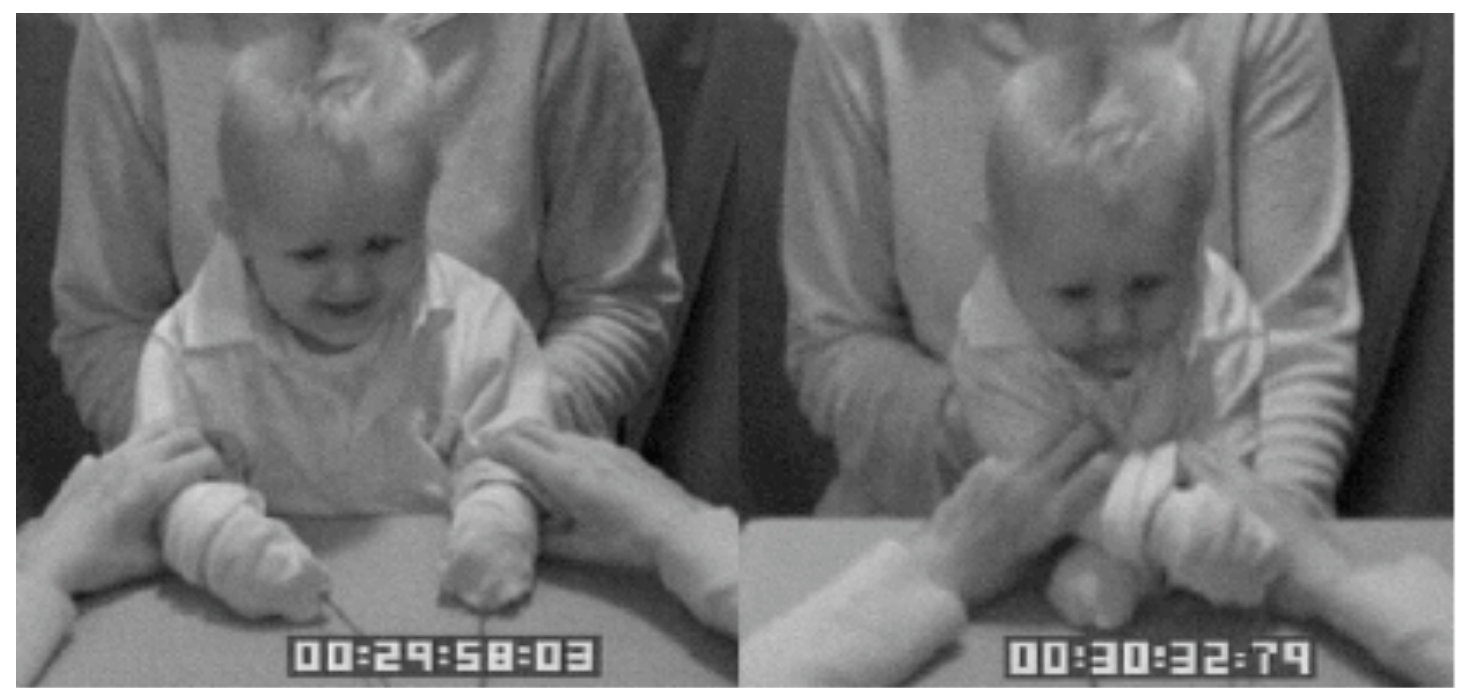


(A) Experiment 1: 10-month-olds

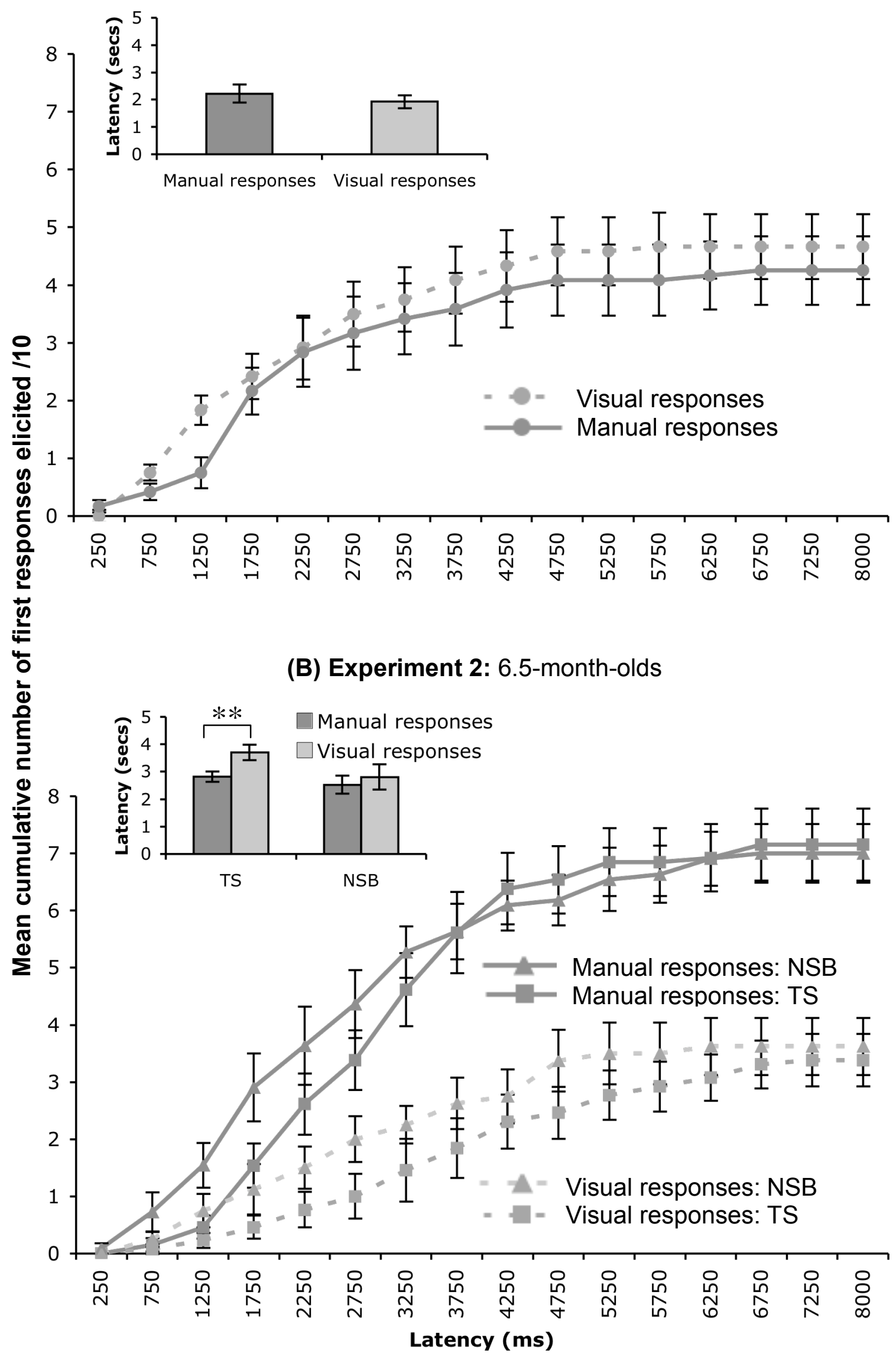

